\title{
Extensive small bowel gangrene at mid-term pregnancy with fruitful outcome
}

\author{
Abdulhadi M. Elbashir ${ }^{1 *}$, Saeed A. Alsareii ${ }^{1}$, Sana B. Mustafa ${ }^{2}$ \\ ${ }^{1}$ Department of Surgery, ${ }^{2}$ Department of Obstetrics and Gynaecology, Faculty of Medicine, Najran University, Najran, \\ KSA
}

Received: 25 March 2017

Accepted: 01 April 2017

\section{*Correspondence:}

Dr. Abdulhadi M. Elbashir,

E-mail: ahadi25@ hotmail.com

Copyright: () the author(s), publisher and licensee Medip Academy. This is an open-access article distributed under the terms of the Creative Commons Attribution Non-Commercial License, which permits unrestricted non-commercial use, distribution, and reproduction in any medium, provided the original work is properly cited.

\begin{abstract}
Intestinal obstruction during pregnancy is very rare. The mechanical intestinal obstruction was mostly due to adhesion from previous surgery. Pregnancy may mask the symptoms of intestinal obstruction, and virgin abdomen adds to the ambiguity of the diagnosis. The mortality and morbidity increased for the mother and the fetus in the presence of bowel gangrene. We present a case of intestinal obstruction at mid-term pregnancy with extensive small bowel gangrene which necessitates right hemicolectomy and ends with a good outcome for the mother and fetus.
\end{abstract}

Keywords: Extensive bowel gangrene, Mid-term pregnancy, Normal delivery

\section{INTRODUCTION}

Several factors often hinder the diagnosis of the acute abdominal problem in a pregnant patient. The tendency to attribute any abdominal symptoms to the pregnancy itself and reluctance to obtain adequate imaging may delay prompt identification and treatment of acute abdomen during pregnancy. ${ }^{1}$ Our patient presentation showed how the delay in diagnosis complicates the condition of the patient. Careful surgical handling may be; life-saving for the mother and the fetus; even after a late presentation.

\section{CASE REPORT}

A twenty-eight years old woman referred from a single doctor hospital complaining of abdominal pain, abdominal distension vomiting, and absolute constipation for three days before presentation. The patient had amenorrhea for five months. She was Para three. Previous deliveries were uneventful. At presentation, the patient was very ill dehydrated febrile and tachypnoeic. Pulse rate was 120/min BP 90/50; RR 28/min. The abdomen was grossly distended. The abdominal wall was edematous. There was guarding tenderness and rebound tenderness all over the abdomen. Bowel sounds were absent per rectal examination revealed an empty rectum. A provisional diagnosis of acute abdomen with the possibility of intestinal obstruction with possible bowel strangulation was made.

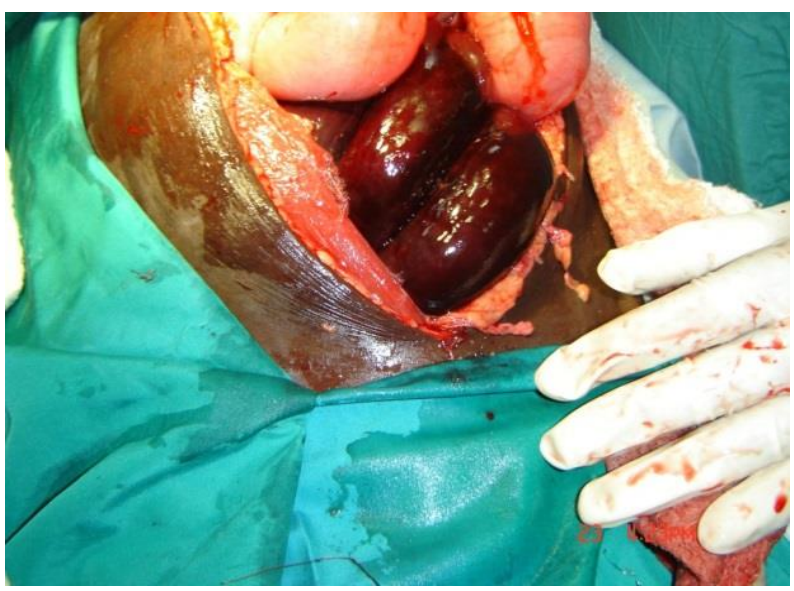

Figure 1: Gangrenous bowel over the gravid uterus. 
Fluid resuscitation and electrolytes correction was carried out, and intravenous antibiotics were started. The condition of the patient was optimized. After six hours of resuscitation, the patient was taken to theater. Laparotomy was performed through a long midline incision. Operative findings were; Oedematous abdominal wall, a gush of hemorrhagic fluid came out and, gangrenous bowel loops were found above the uterus Figure 1.

Extensive gangrene of the ILEAL loops outside the abdomen Figure 2. The ileum was found twisted around a band at the ileocaecal area. Resection of the gangrenous segment and a right hemicolectomy was performed. The manipulations were done carefully; the gravid uterus was not manipulated. Ileo-transverse anastomosis was carried out. The closure was done in layers. Postoperatively Analgesia was given, and antibiotics were continued. The postoperative course was uneventful. The patient was discharged home after seven days in good condition. The patient completed her pregnancy and delivered at term vaginally normal delivery without any complication.

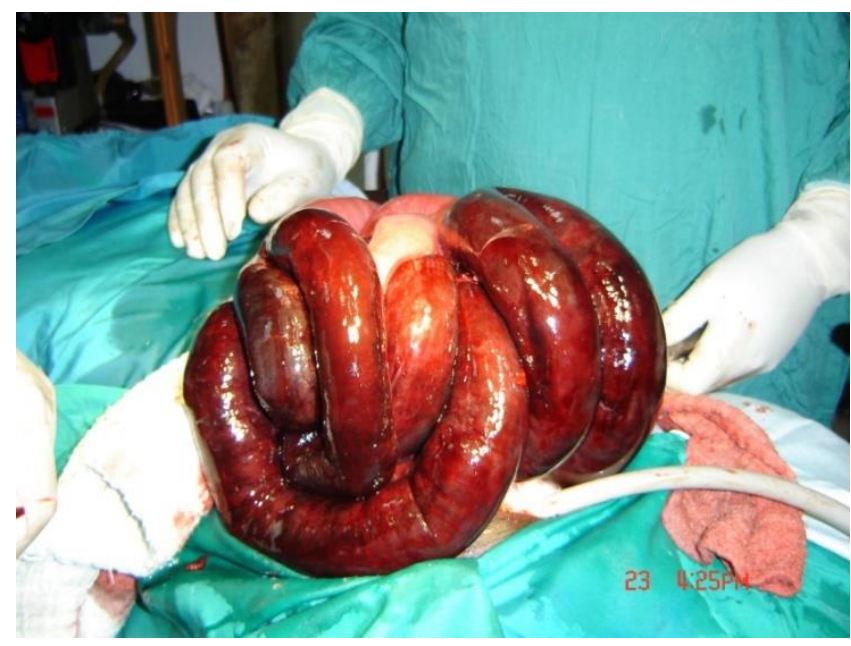

Figure 2: Extensive gangrene of the ILEAL loops outside the abdomen.

\section{DISCUSSION}

Intestinal obstruction is a rare but serious situation during pregnancy, with significant maternal and fetal mortality. The reported incidence varies widely; from 1 in 66,431 to 1 in $1,500 .^{2}$ Adhesions due to previous surgery were reported to be most common causes of mechanical intestinal obstruction complicating pregnancy. ${ }^{3-5}$ In our, patient no history of previous surgery was reported, possibly a congenital band was the reason for the mechanical intestinal obstruction in her case thus the virgin abdomen does not preclude the presence of an intestinal obstruction with pregnancy. ${ }^{6}$ The virgin abdomen adds to the ambiguity of the problem. However, both the mother and fetus are at risk when intestinal obstruction complicates pregnancy; because bowel strangulation, miscarriage rate, and maternal mortality were reported to be very high our patient completed term pregnancy and delivered vaginally. ${ }^{7-9,10}$ Clinical suspicion of the presence, of obstruction and prompt intervention, are required to decrease the morbidity and mortality of this rare complication. In a review of ten cases of bowel obstruction, only one patient had extensive gangrene, in which case the mother and fetus were lost. ${ }^{2}$ Our patient survived and delivered normally. That is to say, meticulous surgical technique and minimal manipulation of the uterus with utmost care might improve the outcome. Uterine manipulations should be kept as little as possible.

Certain anatomic and physiologic changes unique to pregnancy may make the cause of the pain difficult to ascertain. $^{7}$ As the gravid uterus enlarges, it becomes an abdominal organ at around 12 weeks' gestation and compresses the underlying abdominal viscera. This enlargement may make it difficult to localize the pain and may also mask or delay peritoneal signs. ${ }^{7-9}$ We believe this may be the reason for delayed referral of this patient to come late with this extensive bowel gangrene, but luckily enough the mother and the fetus; were saved. Good clinical insight, awareness of the physiologic changes of pregnancy as well as the most common gastrointestinal diseases affecting pregnant women are required for proper management of the pregnant patient. ${ }^{11}$ A careful review of the history, a physical exam performed by keeping the gravid uterus in mind, and well-judged use of radiologic studies mainly MRI are needed for a clear plan for treatment and early intervention in pregnant patients with intestinal obstruction. ${ }^{12}$

\section{CONCLUSION}

The virgin abdomen should not mask the diagnosis of intestinal obstruction during pregnancy. Good management and surgical technique may save the mother and the fetus in intestinal obstruction with pregnancy even in late presentation. Awareness of the doctors and the paramedics' personnel has to be increased about the diagnosis of the acute abdomen during pregnancy to minimize the delay of patients with the critical condition.

Funding: No funding sources Conflict of interest: None declared

Ethical approval: The study was approved by the Institutional Ethics Committee

\section{REFERENCES}

1. Pearce $\mathrm{P}$, Patel H, Vijithan $\mathrm{T}$, Craddock CT, Banerjee S. Gestational intestinal obstruction complicated by cortical blindness-a multidisciplinary case report. JRSM. 2014;5(7):2054270414531120.

2. Chiedozi LC, Ajabor LN, Iweze FI. Small intestinal obstruction in pregnancy and puerperium. Saudi J Gastroenterol. 1999;5(3):134. 
3. Webster PJ, Bailey MA, Wilson J, Burke DA. Small bowel obstruction in pregnancy is a complex surgical problem with a high risk of fetal loss. The Annals of The Royal College Surgeons Eng. 2015;97(5):33944.

4. Vassiliou I, Tympa A, Derpapas M, Kottis G, Vlahos N. Small bowel ischemia due to jejunum volvulus in pregnancy: a case report. Case reports in Obstet Gynecol; 2012.

5. Zachariah SK, Fenn MG. Acute intestinal obstruction complicating pregnancy: diagnosis and surgical management. BMJ; 2014.

6. Latha MS, Abraham R, Chitra TV. Intestinal obstruction in pregnancy: three case reports. Int $\mathrm{J}$ Reprod Contracept Obstet Gynecol. 2013;2:491-3.

7. Dietrich CS, Hill CC, Hueman M. Surgical diseases presenting in pregnancy. Surgical Clinics of North America. 2008;88(2):403-19.

8. Parangi S, Levine D, Henry A, Isakovich N, Pories S. Surgical gastrointestinal disorders during pregnancy. Am J Surg. 2007;193:223-32.
9. Malangoni MA. Gastrointestinal surgery and pregnancy. Gastroenterol Clini North Am. 2003;32(1):181-200.

10. Shnider SM, Webster GM. Maternal and fetal hazards of surgery during pregnancy. Am J Obstet Gynecol. 1965;92:891-900.

11. Alkis I, Kurdoglu M, Kurdoglu Z. Nonobstetric surgical intervention in pregnancy. Eastern Journal of Medicine. 2010;15(1):1.

12. Daimon A, Terai Y, Nagayasu Y, Okamoto A, Sano T, Suzuki Y et al. A case of intestinal obstruction in pregnancy diagnosed by $\mathrm{mri}$ and treated by intravenous hyperalimentation. Case Reports in Obstet Gynecol; 2016.

Cite this article as: Elbashir AM, Alsareii SA, Mustafa SB. Extensive small bowel gangrene at midterm pregnancy with fruitful outcome. Int J Reprod Contracept Obstet Gynecol 2017;6:2095-7. 